\title{
The Topology of Bendless Three-Dimensional Orthogonal Graph Drawing
}

\author{
David Eppstein \\ Computer Science Department, University of California, Irvine \\ eppstein@uci.edu
}

\begin{abstract}
We define an $x y z$ graph to be a spatial embedding of a 3-regular graph such that the edges at each vertex are mutually perpendicular and no three points lie on an axis-parallel line. We describe an equivalence between $x y z$ graphs and 3 -face-colored polyhedral maps, under which bipartiteness of the graph is equivalent to orientability of the map. We show that planar graphs are $x y z$ graphs if and only if they are bipartite, cubic, and three-connected. It is NP-complete to recognize $x y z$ graphs, but we show how to do this in time $O\left(n 2^{n / 2}\right)$.
\end{abstract}

\section{Introduction}

Consider a point set $V$ in $\mathbb{R}^{3}$ (such as the vertices of an axis-aligned cube) with the property that every axis-parallel line in $\mathbb{R}^{3}$ contains either zero or two points of $V$. $V$ forms the vertices of a cubic (that is, 3-regular) graph, in which each vertex $v$ is connected to the other points that lie on the three axis-parallel lines through $v$. We call such a graph an $x y z$ graph. Figure 1 depicts three examples.
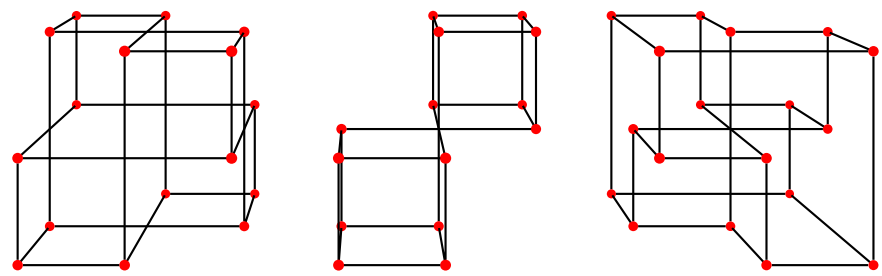

Fig. 1. Three $x y z$ graphs

In contrast to past work on three-dimensional orthogonal drawing with bends [2, 3,6 , 8, 9, 15, 18, 19, 20], an $x y z$ graph provides a simple form of bendless three-dimensional orthogonal drawing. In $x y z$ graphs, edges may cross, but edge crossings may be distinguished visually from vertices by whether the edges stop or pass through them.

In three-dimensional layout of parallel processing intercommunication networks [5], $x y z$ graphs provide a layout in which all connected pairs of processors have an open line of sight between each other. As we show, even-dimensional cube-connected-cycles networks, highly regular graphs used in parallel processing [16], have xyz graph layouts. 
These graphs also have an unexpected connection to topological graph theory and graph coloring: any $x y z$ graph corresponds to a three-coloring of the faces of an embedding of a graph on a 2-manifold. Such face-colored embeddings arise naturally from the GEM (graph-embedded map) representation of manifold embeddings of graphs [4].

In this paper, we prove an equivalence between $x y z$ graphs and certain 3-face-colored cell complexes, which we call $x y z$ surfaces. As we show, an $x y z$ graph is bipartite if and only if the corresponding $x y z$ surface is orientable. We show that it is NP-complete to recognize $x y z$ graphs, and we show how to find $x y z$ graph embeddings in time $O\left(n 2^{n / 2}\right)$; however, planar $x y z$ graphs may be recognized in linear time. Due to space considerations we omit many results, details and proofs; we invite readers to find these in the longer version of this paper at http://arxiv.org/abs/0709.4087.

\section{Topology of $x y z$ Graphs}

If $C$ is a collection of cycles in an undirected graph $G$, we may define a cell complex with a point for each vertex, a line segment for each edge, and a disk for each cycle. For instance, if $G$ is the graph of a cube, and $C$ is the set of four-cycles in $G$, the resulting cell complex consists of the vertices, edges, and facets of a geometric cube. However, complexes may be defined independently of any spatial embedding. If the following conditions are satisfied, the cell complex is a 2-manifold (without boundary) or map:

1. Each edge of $G$ belongs to exactly two cycles of $C$.

2. At each vertex $v$ of $G$, one can reach any incident edge from any other incident edge by a chain of edge-face-edge steps in which each edge and face is incident to $v$.

Cubic graphs automatically satisfy the second condition. The complex defined from $G$ and $C$ is an embedding of $G$ onto a manifold, and the cycles of $C$ are its faces. We define an $x y z$ surface to be an embedding of a cubic graph $G$ with the following properties:

1. Any two faces intersect in either a single edge of $G$ or the empty set.

2. The faces of $C$ can be assigned three colors such that no two faces sharing an edge have the same color.

An embedded graph satisfying the first property is called polyhedral [14]. Polyhedral embeddings of non-cubic graphs may include faces that intersect in a single vertex, but
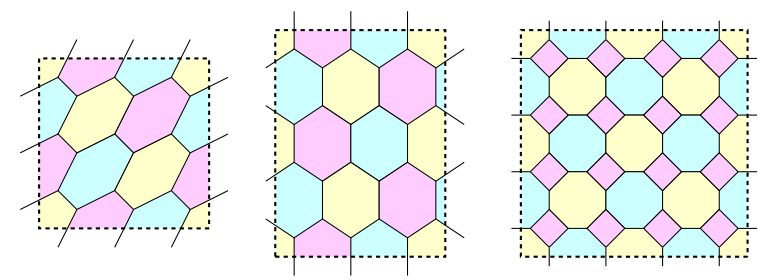

Fig. 2. Three $x y z$ surfaces, each with the topology of the torus. In each case, the torus is depicted as cut and unrolled into a rectangle; the corresponding topological surface is formed by gluing opposite pairs of rectangle edges. 
this cannot happen in a cubic graph. Craft and White [7] study a similar 3-coloring condition on orientable cubic maps without the polyhedral condition.

Theorem 1. $G$ is an xyz graph if and only if $G$ can be embedded as an xyz surface.

Proof. Let $G$ be an $x y z$ graph, and let $C$ consist of the cycles in $G$ that lie in an axisparallel plane. Each edge of $G$ belongs to two such cycles, so, $C$ forms an embedding of $G$ onto a manifold. The cycles of $C$ can be colored according to the coordinate planes they are parallel to. The cycles of $G$ in any single coordinate plane are disjoint, so if two cycles intersect, the intersection must lie on the axis-parallel line formed by the intersection of the two planes containing the cycles, and consists of the edge of $G$ that lies on that same line, fulfilling the requirements of an $x y z$ surface.

Conversely, suppose that $G$ is embedded as an $x y z$ surface, with cycle set $C$. Let $X$, $Y$, and $Z$ be the three color classes of $C$, and let the faces in $C$ be numbered $f_{0}, f_{1}, \ldots$ Each vertex $v$ in $G$ is incident to exactly three faces: $f_{i}$ in $X, f_{j}$ in $Y$, and $f_{k}$ in $Z$ for some $i, j, k$. We assign $v$ the three-dimensional coordinates $(i, j, k)$. If two vertices $u$ and $v$ are adjacent, they share the two coordinates determined by the two faces containing edge $u v$, and lie on an axis-parallel line of the embedding of $G$ into $\mathbb{R}^{3}$. If two vertices are not adjacent, they can lie on at most one face of $C$ and therefore have at most one coordinate in common. Thus, the three axis-parallel lines through each embedded vertex $v$ each contain only $v$ and one of its neighbors so the embedding forms an $x y z$ graph.

The three $x y z$ graphs in Fig. 1 correspond to $x y z$ surfaces that are (left to right) a projective plane resembling the Roman surface, a spherical map combinatorially equivalent to a polyhedron with three hexagonal facets and six quadrilaterals, and an embedding of the Pappus graph on a torus. Figure 2 depicts three $x y z$ surfaces, all tori. The leftmost is the Pappus graph again, the middle surface has 12 faces, 24 vertices, and 36 edges, and the right surface is a torus embedding of the 64-vertex four-dimensional cube-connected cycles network.

Theorem 1 can be used to embed any $x y z$ graph into an $\frac{n}{4} \times \frac{n}{4} \times \frac{n}{4}$ grid: Each face of an $x y z$ graph must have even length, at least four, because it alternates between edges parallel to two coordinate axes. Thus, any color class of an $x y z$ surface coloring has at most $n / 4$ faces: each vertex belongs to one face of that color, but each face contains at least four vertices. Each face provides a value for one of the coordinates in the grid embedding, so the number of distinct values for each coordinate is at most $n / 4$. However this bound is tight only for the cube: any other $x y z$ surface has a face with more than four vertices, and a color class with fewer than $n / 4$ faces, leading to an embedding with fewer than $n / 4$ distinct values in one of the coordinates. For many graphs, permuting the coordinates forms multiple $x y z$ graph embeddings that differ geometrically, although they are combinatorially and topologically equivalent, and smaller grids may sometimes be obtained by using equal coordinate values for multiple faces of the same color. We do not consider problems of choosing coordinate values in order to improve the graph drawing in this paper, but such problems are a natural subject for future work.

As we show in the full version, every $x y z$ graph is triangle-free and 3-vertex-connected. We conclude this section with an interesting connection between bipartiteness and topology. An orientation of a map can be described as a choice of cyclic order on each face of the map such that the two face cycles shared by any edge pass through it in 
opposite directions. A surface is orientable if graphs embedded on it may be oriented; the sphere and torus are orientable, while the projective plane is not.

Theorem 2. Let $G$ be a graph embedded onto an xyz surface. Then $G$ is bipartite if and only if the surface is orientable.

We omit the proof. 2-manifolds may be classified by their orientability and their Euler characteristic $|V|-|E|+|C|$, so by Theorem 2 one may determine the topology of any $x y z$ surface by counting faces and testing bipartiteness.

\section{Algorithms for $x y z$ Embedding}

As we now show, there exist efficient algorithms to determine whether an embedded surface is an $x y z$ surface, or whether a partition of the edges of a graph into three perfect matchings can be used as the three parallel classes of edges in an $x y z$ graph. However, it is not so easy to find an $x y z$ graph representation for an initially unlabeled graph.

Theorem 3. Let $G$ be a connected undirected $n$-vertex graph, and let $C$ be a collection of cycles in $G$. Then in time $O(n)$ we may determine whether $C$ is the set of cycles of an $x y z$ surface embedding of $G$, and if so construct an xyz graph representation of $G$.

Proof. We first check that $G$ is cubic and that $C$ covers each edge of $G$ twice. Next, we assign arbitrary index numbers to the cycles in $C$. Each edge has an associated pair of index numbers, which we order lexicographically. We sort the edges of $G$ according to this lexicographic ordering by two passes of bucket sorting and verify that each consecutive pair of edges in the sorted order has a different pair of faces.

To test 3-colorability of the cycles in $C$, we store a set of the available colors for each cycle (initially, all three colors for each cycle) and a list $L$ of cycles that have only one remaining color. When we color a cycle we remove that color from the available colors of all cycles that share an edge with it, and update $L$ whenever that removal causes an adjacent cycle to have only one remaining available color. We begin by choosing arbitrarily two cycles that share an edge, and assigning arbitrarily two different colors to those two cycles. Then, while $L$ remains nonempty, we remove a cycle from $L$, and assign it the one color that is available to it.

If this process terminates with a 3-coloring of all faces in $C$, we have found an $x y z$ surface representation for $G$. Conversely, suppose that $G$ has an $x y z$ surface representation: we argue that this process will necessarily find a correct 3-coloring of all faces. To show this, permute the colors of the representation if necessary so that they match the colors chosen for the two faces at the start of the algorithm. Every color choice subsequent to that is forced, so the algorithm can neither choose an incorrect color for a face nor eliminate the correct color for any face; the only way it could fail to 3-color all faces would be to terminate with $L$ empty before coloring all faces. But if $f$ is any face of $C$, let $p$ be any path connecting a vertex of the shared edge of the first two colored faces with any vertex of $f$. At any stage in the algorithm until $f$ has been colored, let $v$ be the vertex of $p$ that is closest along the path to the first two colored faces, and that is incident to an uncolored face $f^{\prime}$; then the two differently-colored neighboring faces of $f^{\prime}$ at $v$ would force $f^{\prime}$ to belong to $L$. Thus, $L$ cannot be empty until $f$ is colored, and the algorithm cannot until all faces are colored. 
Corollary 1. Let $G$ be a connected undirected n-vertex graph, and let $E_{1}, E_{2}, E_{3}$ be a partition of the edges of $G$ into three matchings. Then in time $O(n)$ we may determine whether there is an xyz graph representation of $G$ in which $E_{i}$ is the set of edges parallel to the ith coordinate axis.

Proof. For each pair $E_{i}$ and $E_{j}, E_{i} \cup E_{j}$ is a disjoint union of cycles; we let $C$ be the set of cycles formed in this way for all three pairs of matchings, and apply Theorem 3 .

Lemma 1. Let $G$ be a biconnected cubic graph. Then there are at most $2^{(n-2) / 2}$ partitions of the edges of $G$ into three perfect matchings, and these partitions may be listed in time $O\left(2^{n / 2}\right)$.

Proof. We compute an st-numbering of $G$ [12]; that is, an ordering of the vertices of $G$ in which each vertex, except for the ones at the start and the end of the sequence, has a neighbor that occurs earlier in the sequence and a neighbor that occurs later in the sequence. We define a split vertex to be one with one previous neighbor and two later neighbors, and a merge vertex to be one with two previous neighbors and one later neighbor. If there are $k$ split vertices there would be $3+2 k+(n-k-2)$ edges, as the first vertex in the st-numbering is the earlier endpoint of three edges, the split vertices are each the earlier endpoint of two edges, the $n-k-2$ merge vertices are each the earlier endpoint of only one edge, and the final vertex in the $s t$-numbering is the earlier endpoint of no edges. Observing that the graph has $3 n / 2$ edges total and solving for $k$, we find that there must be exactly $(n-2) / 2$ split vertices.

To list all partitions, we then perform a backtracking algorithm in which we assign the edges to partitions in order by their earlier endpoints in the st-numbering; once we make an assignment for an edge $e$ we recursively list all partitions for edges occurring later in this ordering before backtracking and trying an alternative assignment for $e$ (if an alternative exists). If this backtracking process ever reaches a contradictory state in which no possible assignment is available from an edge, it backtracks without recursing.

At the initial vertex of the st-numbering, the backtracking algorithm has no choices to make: it can partition the incident edges into three disjoint subsets in only one way. At the final vertex, there is again no choice to make, because all incident edges must already have been partitioned. And at each merge vertex, there is no choice to make, because there are two incident edges which must already have been placed into two sets of the partition, and the third incident edge can only go in the third set of the partition. Thus, the only branch points of this backtracking algorithm are the split vertices, at which the two edges for which the vertex is the earlier endpoint must be assigned to the two remaining partition sets, in either of two different ways.

Since the algorithm makes a binary choice at each of $(n-2) / 2$ levels of its recursion, its total time is $O\left(2^{n / 2}\right)$. The number of partitions listed is at most the number of leaves in a binary tree of height $(n-2) / 2$, which is $2^{(n-2) / 2}$.

Greg Kuperberg (personal communication) has pointed out that the prisms over $n / 2$ gons form biconnected cubic graphs with $\Omega\left(2^{n / 2}\right)$ partitions into three perfect matchings, showing that this bound is tight to within a constant factor.

Theorem 4. We can test whether a given unlabeled graph is an xyz graph, and if so find an xyz graph representation of it, in time $O\left(n 2^{n / 2}\right)$. 
Proof. We list all partitions into matchings using Lemma 1, and test whether any of them can be used to define an $x y z$ graph representation using Corollary 1.

An implementation of our algorithms for listing all partitions of a cubic graph into perfect matchings and for testing whether a given graph is an $x y z$ graph is available online at http://www.ics.uci.edu/ eppstein/PADS/xyzGraph.py.

\section{Cayley and Symmetric Graphs}

A Cayley graph is a graph having as its vertices the members of a finite group, and its edges determined by a subset of generators for that group; there is an edge from $g$ to $g s$ whenever $g$ is a group element and $s$ is one of the chosen generators. For instance, the cube-connected cycles network $C C C_{n}$, of importance in parallel processing [16], is a Cayley graph for the group of operations on $n$-bit binary words generated by single-bit rotations of the word and flips of the first bit of the word [1]. The cube-connected cycles of order three cannot be an $x y z$ graph, as it is not triangle-free, but we have already seen (Fig. 2, right) that the cube-connected cycles of order four is an $x y z$ graph.

Theorem 5. Let $n$ be any even number greater than or equal to four. Then the cubeconnected cycles network $\mathrm{CCC}_{n}$ is an xyz graph.

We omit the proof. We have not determined whether the cube-connected cycles of odd order greater than three may be an $x y z$ graph.

Another important cubic Cayley graph is that of the symmetric group of permutations on four elements, generated by transpositions of adjacent elements. This graph forms the skeleton permutohedron, the convex hull of the 24 permutations of $(1,2,3,4)$ in the three-dimensional subspace $x+y+z+w=10$ of $\mathbb{R}^{4}$ [13]. Moving each permutation to the position of its inverse causes the edges to fall into three parallel classes, and if we then transform the drawing affinely so that these three classes are perpendicular, the result is an $x y z$ graph. Figure 3 shows the permutohedron, the resulting $x y z$ drawing, and another $x y z$ drawing in which we have permuted the coordinate values manually to reduce the number of crossings. A different Cayley graph for the same symmetric group, generated by the permutations $(12)(3)(4),(13)(2)(4)$, and $(14)(2)(3)$, is the 24 -vertex symmetric graph shown in Fig. 5. Higher dimensional permutohedra have too many edges per vertex to be xyz graphs, but a different Cayley graph for the symmetric group $S_{n}$, generated by a permutation that swaps the first two elements and another permutation that rotates all but the first element, is an $x y z$ graph whenever $n$ is an odd number greater than three. For $n=5$ this graph forms the skeleton of a uniform polyhedron, the truncated dodecadodecahedron (Fig. 4), which has 30 square faces, 12 decagonal faces, and 12 star-shaped faces with ten vertices per face, interpenetrating each other to form a complex surface. The 3-face-coloring by which the truncated dodecadodecahedron can be recognized as an xyz surface coincides with the partition of its faces into different shapes.

Next, consider the points $(x, y, z)$ in the $k \times k \times k$ grid for which $x+y+z$ is 0 or 1 (mod $k$ ). They form an $x y z$ graph that is symmetric: that is, its symmetries act transitively on incident vertex-edge pairs. For $k=3$ this produces the Pappus graph. The graph 

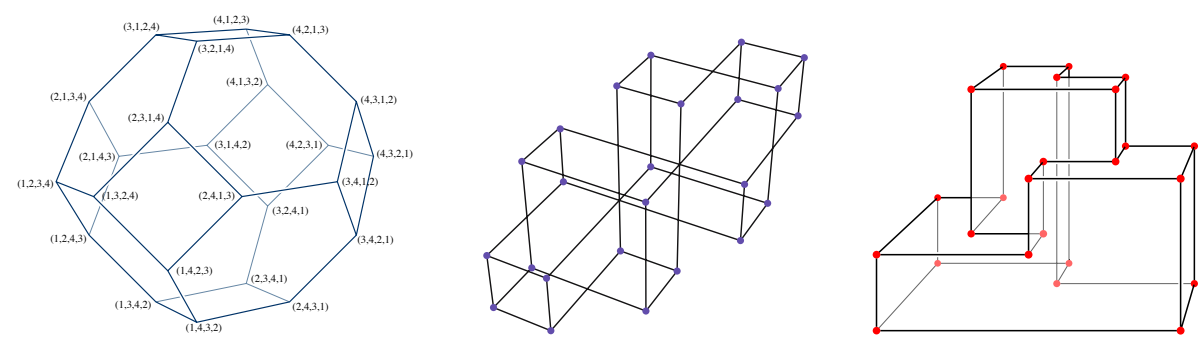

Fig. 3. The permutohedron (left) and two $x y z$ drawings of the corresponding Cayley graph (center and right). The center drawing is formed by connecting pairs of permutohedron vertices that differ by swapping consecutive coordinates and affinely transforming so that the edges are perpendicular; the right drawing permutes the values of each coordinate to realize the Cayley graph as the skeleton of an orthogonal polyhedron.

$F_{32}$ shown on the left of Fig. 5 is the Dyck graph, a 32-vertex symmetric cubic graph embedded by the same construction with $k=4$. (Here $F_{n}$ refers to the unique $n$-vertex cubic symmetric graph as listed in the Foster census [17].) Visible near the equatorial plane of the Dyck graph drawing are a number of six-vertex cycles that are not faces of the corresponding $x y z$ surface (they use edges parallel to all three coordinate axes, while the surface faces are restricted to axis-parallel planes); this pattern persists for larger $k$, and if one analogously forms an infinite $x y z$ graph from the points in a threedimensional grid with coordinates summing to 0 or 1 , the result is isomorphic as a graph to the hexagonal tiling of the plane [11].

A different construction for cubic symmetric $x y z$ graphs is possible, based on the infinite tiling of the plane by regular hexagons. Three-color the hexagons of this tiling, choose a rhombus with angles of $\pi / 3$ and $2 \pi / 3$, having its vertices at the centers of tiles that are all the same color, and form a torus by gluing opposite sides of this rhombus together. The result, as shown in Fig. 5, center, is an $x y z$ surface. The graph embedded on this surface is symmetric, because we can transform any incident vertex-edge pair into any other such pair by a combination of translations and rotations by an angle of $\pi / 3$. When $n=18 q^{2}$ for some $q$, one can form an $n$-vertex symmetric graph using both of the constructions above, either by forming a torus from a rhombus containing $n / 2$ hexagons, with sides parallel to the edges of the hexagonal tiling, or by using the points congruent to 0 or 1 in a $3 q \times 3 q \times 3 q$ grid. Both graphs formed in this way are isomorphic, but (except for $k=1$ ) the $x y z$ graph embeddings resulting from these constructions are inequivalent: the $x y z$ surface resulting from the $k \times k \times k$ grid has fewer faces with more vertices per face. For instance the 72-vertex cubic symmetric graph $F_{72}$ forms an $x y z$ surface with 18 12-vertex faces (a $6 \times 6 \times 6$ grid) or with 366 -vertex faces (a rhombus containing 36 hexagons).

Figure 5, right, shows another cubic symmetric graph, $F_{40}$, that does not fit into either of these constructions. $F_{40}$ is the double cover of the regular dodecahedron; that is, it is the bipartite graph formed by making two copies of each dodecahedron vertex, colored black and white, and connecting the white copy of each vertex to the black copy of each of its neighbors. Its $x y z$ graph representation has faces of three types: two decagons formed as the double covers of a pair of opposite dodecahedron faces, two 


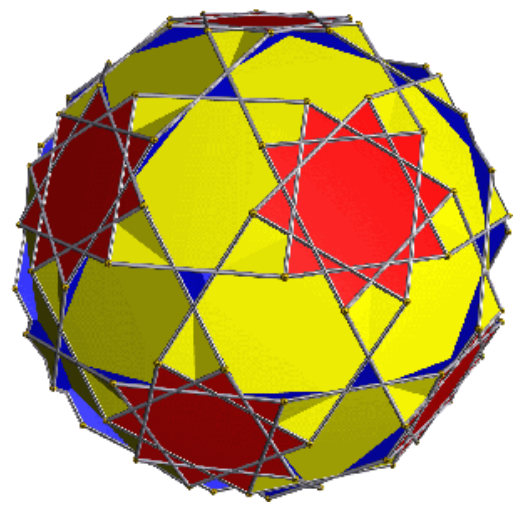

Fig. 4. The truncated dodecadodecahedron, from Wikimedia Commons, originally uploaded to Wikipedia by Tom Ruen in October 2005 and created using Robert Webb's Great Stella software (http://www.software3d.com/Stella.html). The vertices and edges of this shape form a Cayley graph for the symmetric group $S_{5}$, with generators (12)(3)(4)(5) and (1)(2345); the faces in the figure are 3-colored, giving an $x y z$ surface representation of the graph.
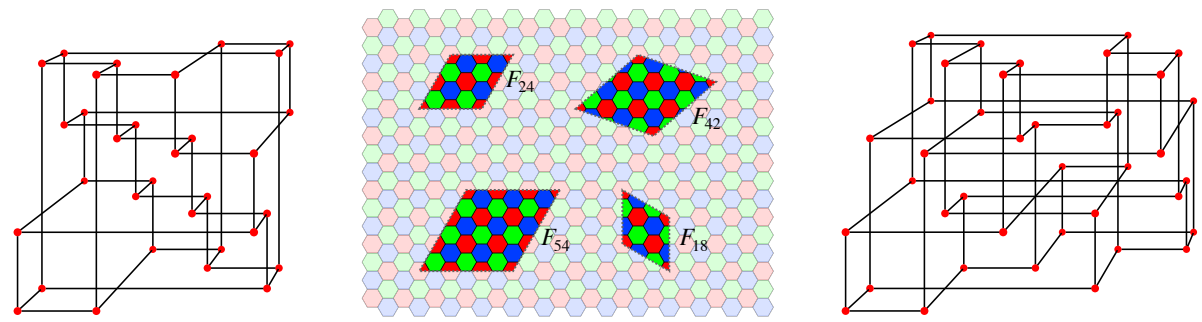

Fig. 5. Left and right: the Dyck graph $F_{32}$ and the double cover of the dodecahedron $F_{40}$, two cubic symmetric graphs drawn as $x y z$ graphs. Center: Construction of cubic $x y z$ surfaces as toric quotients of the three-colored hexagonal tiling.

more decagons formed from the double cover of the equator between those two faces, and ten octagons formed as the boundary of a pair of adjacent dodecahedron faces that lie on opposite sides of the equator. There are six ways of choosing two opposite faces from which the decagons are formed, and once that choice is made there remain two ways of choosing the octagons to form an $x y z$ surface, so $F_{40}$, viewed as a labeled graph, has 12 combinatorially distinct $x y z$ surface representations.

We applied our implementation of an $x y z$ graph embedding algorithm to the Foster census of symmetric cubic graphs [17] and did not find any other $x y z$ graphs of this type on 56 or fewer vertices.

\section{Planar and Nonplanar Graphs}

We may exactly characterize the planar $x y z$ graphs. 

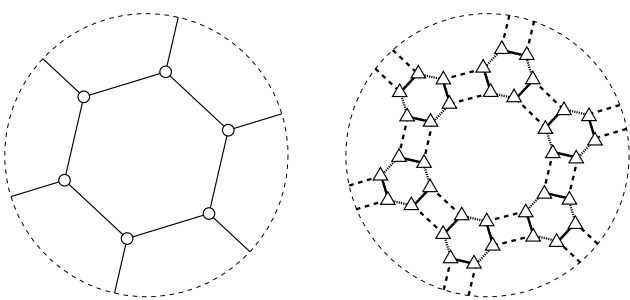

Fig. 6. An embedding of $K_{3,3}$ in the projective plane with one hexagonal face and three quadrilateral faces (left) and a GEM representation of the embedding (right). From [10].

Theorem 6. Let $G$ be a planar graph. Then $G$ is an xyz graph if and only if $G$ is bipartite, cubic, and 3-connected. If it is an xyz graph it has a unique representation as an xyz surface, up to permutation of the face colors of the surface.

Corollary 2. We may test in linear time whether a planar graph $G$ is an xyz graph.

The $x y z$ graph formed from the points $(x, y, z)$ in the $k \times k \times k$ grid for which $x+y+z$ is 0 or $1(\bmod k)$ has $2 k^{2}$ vertices and $3 k^{2}$ edges but only $3 k$ faces (one per axis-aligned plane) so its Euler characteristic is $3 k-k^{2}$. If $G$ and $G^{\prime}$ are $x y z$ graphs, with designated vertices $v$ and $v^{\prime}$, we may form the connected sum of $G$ and $G^{\prime}$ by aligning the two graphs in $\mathbb{R}^{3}$ so that $v$ and $v^{\prime}$ coincide (and so that no pairs of vertices, one from $G$ and one from $G^{\prime}$, lie on an axis-parallel line unless both vertices in the pair are adjacent to $v$ and $v^{\prime}$ ) and then by removing $v$ and $v^{\prime}$, leaving in their place a non-vertex point where the lines through three edges cross. The 14-vertex planar graph in the center of Fig. 11 can be viewed in this way as a connected sum of two cubes. In terms of $x y z$ surfaces, the connected sum operation can be viewed as cutting the two surfaces by a small disk surrounding each of $v$ and $v^{\prime}$, and gluing the three faces surrounding this hole on one surface to the faces of corresponding colors surrounding the hole on the other surface, to form a handle connecting the two surfaces. By forming connected sums of tori and projective planes (the $x y z$ graphs on the left and right of Fig. 1 1 respectively), we may form $x y z$ surfaces of any topological type.

An alternative construction allows arbitrary surfaces to be represented as $x y z$ surfaces: the graph encoded map (GEM, Fig. 6) [4, 10]. Let $G$ be any graph embedded on a 2-manifold in such a way that each face of the embedding is a topological disk bounded by a simple cycle of $G$. A flag of this embedding is a triple of a vertex, edge, and face that are all incident to each other, and the graph encoded map $M$ of this embedding is a 3-edge-colored cubic graph, having a vertex for each flag of the embedding of $G$. Two vertices of $M$ are adjacent if the corresponding two flags differ only in a vertex, differ only in an edge, or differ only in a face; the edge coloring of $M$ determines which type of difference each edge of $M$ represents. $M$ itself can be embedded on the same surface, with a $2 k$-cycle for each vertex of degree $k$ in $G$ or each face in $G$ that is surrounded by $k$ edges, and a 4-cycle for each edge of $G$. These cycles form an $x y z$ surface, in which the color of a face in the GEM is determined by whether it represents a vertex, face, or edge in $G$, so $M$ is an $x y z$ graph. 

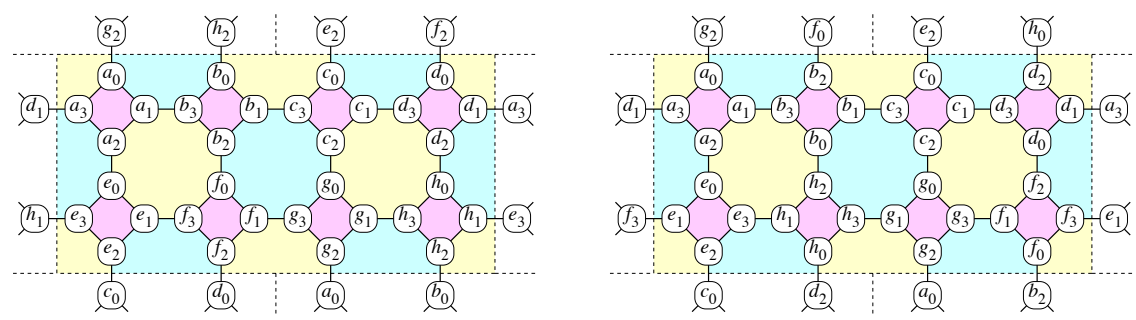

Fig. 7. A graph that can be embedded on the torus as an $x y z$ surface in two different ways

The 32-vertex graph shown in Fig. 7 has two different torus embeddings, showing that the uniqueness of $x y z$ surface representations for planar graphs does not directly generalize to other surfaces. The colored region of the figure shows a rectangle that can be glued to itself in a brick wall pattern to form a torus; vertices are repeated outside the colored rectangle to show the graph edges that cross the glued rectangle boundaries. This ambiguously-embeddable graph plays a key role in our NP-completeness proof in Sect. 6

\section{Complexity of $x y z$ Graph Recognition}

We show that recognizing $x y z$ graphs is NP-complete, via a reduction from graph 3colorability, using pieces of surfaces to represent the vertices to be 3-colored and the edges that connect them; these pieces are linked together using connected sum operations. The edge gadget is based on two copies of the graph of Fig. 7 connected to each other and the vertex gadgets by narrow tubes. We represent the choice of a color for a vertex by the choice of which coordinate axis to make parallel to certain edges of the vertex gadget. We omit the details for lack of space.

Theorem 7. It is NP-complete, given an undirected graph $G$, to determine whether $G$ can be represented as an xyz graph.

\section{Conclusions}

We have studied examples, algorithms, topology, and complexity of $x y z$ graph drawing. Our investigation opens up several avenues for further research:

- In our construction of an $x y z$ graph from an $x y z$ surface, we may permute the coordinate values associated with each face, giving drawings with different appearances for a single $x y z$ surface representation (Fig. 3). How difficult is it, given an $x y z$ surface, to find a permutation of coordinate values that minimizes the number of crossings?

- In some cases it may be possible to reduce the volume of the grid into which an $x y z$ graph is embedded by allowing multiple faces of an $x y z$ surface to share the same coordinate value. How difficult is it to find the minimum volume $x y z$ graph drawing of a given $x y z$ surface? 


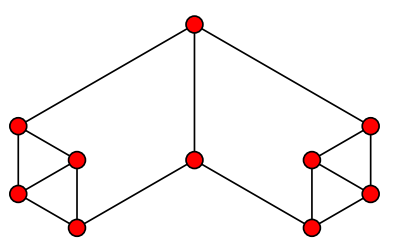

Fig. 8. A point set such that lines in three parallel families each contain zero or exactly two points, and the cubic graph derived from it. This graph is not an $x y z$ graph, as it contains triangles.

- The rightmost drawing of the permutohedron in Fig. 3 shows it as the boundary of an orthogonal polyhedron (perhaps suitable for the design of a building). How difficult is it to determine whether such a representation exists for a given bipartite 3-connected cubic planar graph?

- Our reduction from graph coloring to $x y z$ graph recognition produces graphs of high genus, but recognizing $x y z$ graphs is easy in the case of genus zero (planar graphs). Is there an efficient algorithm for recognizing $x y z$ graphs of bounded genus?

- For Cayley graphs with one self-inverse and one non-self-inverse generator, the difficulty in finding $x y z$ graph representations is linked to the need to independently orient each cycle formed by the non-self-inverse generator. However, as these graphs are highly symmetric, it seems natural to hope that these cycles may be oriented in a symmetric way that avoids the need for testing all orientations of all cycles. Is there a Cayley graph that may be represented as an $x y z$ graph only by orienting its cycles asymmetrically?

- Kuperberg's example of the prism shows that our algorithm for testing $x y z$ graph representability using all partitions of the graph into three matchings cannot be improved, unless we avoid some partitions. However, for the prism itself, there are many partitions that can safely be avoided: for an $x y z$ graph representation, we cannot use any partition into three matchings that uses three different orientations in a single quadrilateral. One can also devise similar conditions that restrict the matchings in hexagons and other short cycles of a given graph. Can one take advantage of these forbidden configurations to eliminate some partitions into matchings earlier in the algorithm and reduce its running time?

- In our discussion of graphs represented by the points with coordinates summing to 0 or 1 in a $k \times k \times k$ grid, we briefly referred to a similar construction of an infinite $x y z$ graph in an infinite three-dimensional grid, isomorphic to the hexagonal tiling of the plane, a graph treated in more detail in another paper [11]. To what extent can the correspondence between $x y z$ graphs and $x y z$ surfaces be generalized to infinite graphs? What is the most appropriate way of handling the infinite chains of edges parallel to a single coordinate plane that can arise in the infinite case?

- If a planar point set intersects any line parallel to the sides of an equilateral triangle in either zero or two points, we may define a cubic graph from it analogously to the three-dimensional definition of $x y z$ graphs; any $x y z$ graph has a planar projection of this type. However, these planar three-orientation graphs are more general than $x y z$ graphs; Fig. 8 shows a graph of this type that is not an $x y z$ graph. To what extent may our theory be extended to these graphs? 


\section{Acknowledgements}

We thank Ed Pegg, Jr., Tomo Pisanski, Frank Ruskey, Tom Tucker, Arthur White, and the anonymous reviewers for Graph Drawing 2008 for helpful comments. Work supported in part by NSF grant 0830403 . Except as noted, all figures in this paper are by the author; all figures remain the copyright of their creators and are used by permission.

\section{References}

1. Annexstein, F., Baumslag, M., Rosenberg, A.L.: Group action graphs and parallel architectures. SIAM J. Comput. 19(3), 544-569 (1990)

2. Biedl, T., Shermer, T.C., Whitesides, S., Wismath, S.K.: Bounds for orthogonal 3-D graph drawing. J. Graph Alg. Appl. 3(4), 63-79 (1999)

3. Biedl, T., Thiele, T., Wood, D.R.: Three-dimensional orthogonal graph drawing with optimal volume. Algorithmica 44(3), 233-255 (2006)

4. Bonnington, C.P., Little, C.H.C.: The Foundations of Topological Graph Theory. Springer, Heidelberg (1995)

5. Calamoneri, T., Massini, A.: Optimal three-dimensional layout of interconnection networks. Theor. Comput. Sci. 255(1-2), 263-279 (2001)

6. Closson, M., Gartshore, S., Johansen, J.R., Wismath, S.K.: Fully dynamic 3-dimensional orthogonal graph drawing. J. Graph Alg. Appl. 5(2), 1-34 (2001)

7. Craft, D.L., White, A.T.: 3-maps. Discrete Math. (2008)

8. Eades, P., Stirk, C., Whitesides, S.: The techniques of Komolgorov and Bardzin for threedimensional orthogonal graph drawings. Inf. Proc. Lett. 60(2), 97-103 (1996)

9. Eades, P., Symvonis, A., Whitesides, S.: Two algorithms for three dimensional orthogonal graph drawing. In: North, S.C. (ed.) GD 1996. LNCS, vol. 1190, pp. 139-154. Springer, Heidelberg (1997)

10. Eppstein, D.: Dynamic generators of topologically embedded graphs. In: Proc. 14th Symp. Discrete Algorithms, pp. 599-608. ACM and SIAM (January 2003)

11. Eppstein, D.: Isometric diamond subgraphs. In: Proc. 16th Int. Symp. Graph Drawing (2008)

12. Even, S., Tarjan, R.E.: Computing an st-numbering. Theor. Comput. Sci. 2(3), 339-344 (1976)

13. Gaiha, P., Gupta, S.K.: Adjacent vertices on a permutohedron. SIAM J. Appl. Math. 32(2), 323-327 (1977)

14. Kochol, M.: 3-regular non 3-edge-colorable graphs with polyhedral embeddings in orientable surfaces. In: Proc. 16th Int. Symp. Graph Drawing (2008)

15. Papakostas, A., Tollis, I.G.: Algorithms for incremental orthogonal graph drawing in three dimensions. J. Graph Alg. Appl. 3(4), 81-115 (1999)

16. Preparata, F.P., Vuillemin, J.: The cube-connected cycles: a versatile network for parallel computation. Commun. ACM 24(5), 300-309 (1981)

17. Royle, G., Conder, M., McKay, B., Dobscanyi, P.: Cubic symmetric graphs (The Foster Census). Web page (2001), http://people.csse.uwa.edu.au/gordon/remote/foster/

18. Wood, D.R.: An algorithm for three-dimensional orthogonal graph drawing. In: Whitesides, S.H. (ed.) GD 1998. LNCS, vol. 1547, pp. 332-346. Springer, Heidelberg (1999)

19. Wood, D.R.: Bounded degree book embeddings and three-dimensional orthogonal graph drawing. In: Mutzel, P., Jünger, M., Leipert, S. (eds.) GD 2001. LNCS, vol. 2265, pp. 312327. Springer, Heidelberg (2002)

20. Wood, D.R.: Optimal three-dimensional orthogonal graph drawing in the general position model. Theor. Comput. Sci. 299(1-3), 151-178 (2003) 